\title{
EL LÉXICO DEL PROTOCOLO
}

\author{
María Luz Álvarez Rodríguez
}

Investigadora. Universidad de Vigo.

\begin{abstract}
Protocol and ceremonial lexicon is the problem that we consider in this article because, in addition to not being known in general by the society, also it isn't between their own professionals and academicians. Few persons know the terms of this subjetc and especially, few people know what is the meaning of the mentioned terms. But unfortunately protocol authors have this problem that is demonstrated in their books. With the purpose of announcing the protocol subject to the spanish academic field, the present article considers to know the protocol terminology in Spain across his bibliography to place his theoretical bases and to impel his development in our country.

\section{Resumen}

El léxico del protocolo y el ceremonial es el problema que nos planteamos en este artículo ya que, además de ser en general desconocido por la sociedad, también lo es entre sus propios profesionales y académicos. Pocas personas conocen los términos de esta materia y sobre todo, poca gente sabe cuál es el significado de dichos términos. Pero lamentablemente los autores del protocolo tienen esta problemática que se evidencia en sus obras. Con la finalidad de dar a conocer en el ámbito científico español al protocolo, este artículo se plantea conocer la terminología del protocolo en España a través de su bibliografía para asentar sus bases teóricas e impulsar su desarrollo en nuestro país.
\end{abstract}

\section{Palabras clave}

Protocolo - Ceremonial - Comunicación - Léxico - Terminología - España

\section{Key words}

Protocol - Ceremonial - Communication - Lexicon - Terminology - Spain 


\section{Introducción}

El significado del protocolo es un gran desconocido en España. En el día a día se refleja continuamente la falta de conocimiento que tiene la sociedad española sobre esta materia. Periodistas, políticos, ciudadanos... dan muchos ejemplos de ello al emplear incorrectamente el léxico propio del protocolo (Álvarez, 2008c). Pero lo más grave es que incluso entre sus profesionales y académicos falta acuerdo nocional. Simplemente podemos poner como ejemplo la confusión existente a la hora de denominar la propia materia que puede ser: “Ceremonial y Protocolo", “Ceremonial” o "Protocolo". Esto, que en principio resulta tan banal, supone en esta materia un problema ya que existe una extendida confusión a la hora de emplear y definir su propio léxico.

\section{Objetivos}

El objetivo de este artículo es ofrecer un análisis atento, riguroso y profundo en la medida de lo posible del léxico del protocolo, siendo una cuestión pendiente y original entre los trabajos académicos españoles de protocolo. Este estudio persigue indicar el léxico clave del protocolo establecido por sus autoridades, cuáles son las palabras más empleadas en esta materia y cuáles son los significados de dichas palabras, lo que nos permitirá conocer mejor la materia a través de su terminología específica.

\section{Metodología}

Sustentándonos en el marco teórico de la comunicación, para la realización de este estudio teórico o básico (Saperas, 1998) se ha consultado la bibliografía completa del protocolo y ceremonial publicada en España por autores españoles hasta el año 2006, mediante múltiples técnicas investigadoras como son: el análisis documental, el análisis textual, el análisis de contenido y el análisis del discurso. Por lo tanto el estudio tiene una carga, tanto analítica como interpretativa, al emplear técnicas cualitativas y cuantitativas para realizar la revisión científica de la casi totalidad de la literatura bibliográfica de la materia. Ello implica una selección, clasificación y síntesis de los recursos terminológicos básicos del protocolo en España elaborados por un conjunto de autores que construyen el capital intelectual y experiencial de esta materia en sus monografías (Álvarez, 2008b). De este modo, por un lado pretendemos ofrecer reunidos materiales hasta el momento dispersos, al mismo tiempo que construimos la expresión empírica de los calificativos definidores del protocolo a partir de la observación indirecta de su realidad mediante análisis documentales de su bibliografía en España. 


\section{El problema terminológico del protocolo}

El protocolo presenta un grave problema terminológico. Las palabras empleadas por este materia son totalmente desconocidas entre la sociedad, pero también entre sus profesionales y académicos que ni siquiera se ponen de acuerdo a la hora de establecer un término claro para denominarla.

La causa principal de esta ignorancia terminológica se puede entrever en estas palabras de Marín (2000: 35):

Hasta hace poco tiempo, los términos ceremonial y protocolo eran empleados con asiduidad sólo por un restringido número de personas y reservado su uso estrictamente al ámbito de los actos oficiales de Estado de las relaciones diplomáticas e intergubernamentales y en el marco de determinadas instituciones o corporaciones. Sin embargo, hoy en día están presentes en todos los estamentos y grupos sociales y son utilizados con relativa frecuencia por las personas en sus celebraciones privadas. Las causas que han impulsado este cambio han sido en esencia: el incremento de las relaciones interestatales, la creciente importancia y proliferación de las organizaciones internacionales -gubernamentales o no-, la mayor presencia social de instituciones públicas y privadas y la gran influencia que tienen las empresas y otras organizaciones en el desarrollo socioeconómico actual.

Por lo tanto, las palabras del protocolo y el ceremonial, las grandes desconocidas hace unos años y limitadas a un círculo social cerrado, son ya algo habitual en la sociedad, reclamando su lugar. Pero el traslado de dicho léxico a toda la sociedad ha causado, de momento, bastantes problemas ya que su significado se malinterpreta con frecuencia.

Y lo más destacable es que, incluso entre los propios autores de esta materia, se observa una falta de criterio y grandes ambigüedades a la hora de definir las palabras más básicas como son protocolo y ceremonial. Eso demuestra dificultades para poder afrontar algo elemental -que no fácil- para cualquier ciencia como es el establecimiento de una terminología propia.

Esta situación la consideramos grave ya que los conceptos son componentes tan indispensables del método científico como las propias hipótesis; es más, éstas no pueden existir sin aquellos. El sistema teórico de la ciencia es un sistema conceptual y si el protocolo no tiene sus propios conceptos, no podrá tener teoría y muchos menos ser considerado una ciencia propia o auxiliar.

A continuación podemos observar como varias autoridades del protocolo comentan en sus obras esta problemática a la que todavía no se le ha encontrado solución pero de la que queremos dejar constancia. La autoridad más clara a la hora de abordar este tema es Francisco López-Nieto (2000a, I: 15). 
Observaba el pensador estoico Epicteto que el principio de toda doctrina debía ser la consideración o el estudio de su nombre, respecto a lo cual añadía Mendizábal que, a la verdad, examinar la significación de los términos suele ayudar no poco a descubrir la naturaleza de las cosas por ellos presentadas. Y esta conveniencia, o necesidad, se hace más sensible cuando de cuestiones protocolo se habla. Primero, porque en ésta materia hasta ahora poco tratada, tanto doctrinal como legalmente, y casi siempre desde un punto de vista práctico, sin una elaboración teórica y sistemática. Y segundo, porque ese modesto tratamiento se ha producido, por lo general, utilizando una terminología confusa y poco definida. Ésta es la razón de que nuestras palabras iniciales vayan enderezadas a establecer unos conceptos previos que estimo necesarios para acometer la tarea. Cuando la legislación, tanto la antigua como la vigente, se ha enfrentado con la conveniencia de ordenar esta materia, ha utilizado terminología muy varia y no siempre sobrada de nitidez, cuando no de carácter ambivalente. Si ante la ausencia de definiciones legales, nos atenemos a las descripciones semánticas que el Diccionario de la Academia de la Lengua nos ofrece, observamos que unas mismas voces pueden ser utilizadas a través de acepciones diversas y que con frecuencia el contenido de los conceptos reitera. Tal ocurre con los vocablos distinción, dignidad, título, tratamiento, honor, ceremonial, etiqueta y protocolo.

Carlos Fuente también comparte la idea sobre el DRAE (2004b: 30):

Como puede desprenderse de estas expresiones del Diccionario de la Real Academia Española, pocas conclusiones clarificadoras podemos extraer y aunque nos conduzcan a una ruptura con la máxima autoridad lingüística de nuestro país, lo cierto es que no vemos representado en sus significaciones lo que realmente es hoy el Protocolo, el Ceremonial y la Etiqueta. Otros diccionarios de prestigio van un poco más lejos y aportan algunas luces aunque demasiadas pocas en tanta sombra.

Además Fuente refleja la situación de la terminología en la sociedad española (2004b: 17);

Muchos medios de comunicación, cuando tratan el tema, llevan la cuestión protocolaria al arte de colocar la mesa, el orden de entrada de los novios en el cortejo o el momento adecuado de enviar las flores a tu secretaria en el día de su parto. La sociedad general desarrollada reclama insistentemente, por su parte, un mayor esfuerzo en la formación de nuestros hijos en el ámbito de los buenos modales, habiendo en este contexto un erróneo sentido en la deseada vuelta atrás de la asignatura de Urbanidad. En su terreno, los profesionales reivindicamos un espacio de profesión, entendiendo el Protocolo como el arte de la organización y a su profesional como un gestor de la organización de los actos en las instituciones públicas y privadas.

Por su parte María Teresa Otero explica la problemática de esta materia a través de la teoría (2000: 127): 
Son muchas las definiciones de protocolo realizadas hasta el momento, pero no son generalmente demasiado sistemáticas. La utilización de este término es bastante reciente, generalizándose a partir de la segunda mitad de este siglo, y anteriormente aparecen siempre los términos «ceremonial» $\mathrm{y}$ «etiqueta». Cuando encontramos modernamente alguna definición de protocolo suele estar tomada de algún texto anterior o se refiere a aspectos parciales de la materia, y aún en las obras más actuales puede apreciarse cierta confusión entre estos conceptos, fruto tal vez del escaso interés teórico que ha originado su investigación teórica, frente a la gran importancia de su desarrollo práctico y profesional. Si bien hay abundantes documentos históricos que dan testimonio del ceremonial existente en las más distintas culturas y de la etiqueta en las distintas cortes, no es hasta finales del siglo XIX cuando comienza a aparecer la palabra protocolo en los textos con un sentido aproximado al de hoy.

Y también deja claro sus deseos para el futuro de esta materia (Otero, 2001: 20-21):

Nos gustaría expresar nuestro deseo de que se continuara profundizando en la teoría del ceremonial y el protocolo y sus relaciones con la comunicación no verbal, con el objeto de que investigadores, docentes y profesionales de estas materias, unificáramos criterios a la hora de utilizar la terminología adecuada para referirnos a ellas. Sería igualmente de gran interés que se enriqueciera el vocabulario de los excelentes ceremonialistas y protocolistas existentes en nuestro país, y que se comenzaran a utilizar con propiedad términos como orden de precedencias, jerarquización, ordenación interna de cargos, plano de mesa, etiqueta en el vestido o ubicación de comensales, eludiendo el uso general e indiscriminado de la palabra «protocolo» para cualquier acción que implique orden en el contexto de las relaciones sociales.

Todo esto nos obliga a obviar la lexicografía de las enciclopedias y diccionarios realizados por expertos lingüistas acerca del tema protocolo, ya que no han conseguido solucionar la problemática terminológica existente, y al mismo tiempo nos obliga a investigar la propia terminología y definiciones empleadas por las autoridades de esta materia en sus textos, para así aproximarnos más a la auténtica base del protocolo en España que todavía no está constituida, aunque cada vez hay más intentos (Otero, 2000; Hernández, 2006; Sierra, 2007; Álvarez, 2008a).

\section{Investigación}

Expuesta ya la problemática terminológica del protocolo y el ceremonial vamos a explicar cual será nuestra investigación. Investigación terminológica que no va a seguir la teoría textual ni lingüística a 
la hora de desarrollarse ya que, no somos lingüistas, ni nos interesa hacer un estudio lingüístico. Recordamos además que esta situación, que parece exclusiva de esa materia, es necesaria en todas las ciencias en orden a sentar las bases de su fundamentación donde destaque su dimensión estructural. Pretendemos por tanto, una aproximación a los significados de ciertos términos aproximándonos a su vez al cuerpo fundante del protocolo que nos permitirá un estudio teórico como el desarrollado por Daniel Martí en otro artículo de este mismo número.

Tras considerar diversas opciones determinamos que la herramienta más eficaz para llevarlo a cabo este estudio es un Glosario, considerándolo como: un catálogo de palabras de una misma materia, de un mismo campo de estudio, etc., definidas o comentadas. Por lo que, partiendo de dicha definición, concluimos que tras finalizar un glosario, tendremos una lista científica de palabras relacionadas directamente con el protocolo, con un significado claro, que nos permitirá dos cosas: su uso práctico a la hora de estudiar y hablar sobre protocolo, y el establecimiento de una base científica para una posterior elaboración de un cuerpo teórico del protocolo.

Pero para poder hacer ese Glosario científico tenemos que realizar previamente varios estudios objetivos de carácter cuantitativo y cualitativo.

Primero buscamos la existencia de Glosarios científicos de protocolo que nos puedan servir de ejemplo para nuestro estudio, pero constatamos que ningún autor creó ningún Glosario científico, aunque hay que señalar que sí vemos algunos catálogos de palabras similares a un Glosario en dos obras de Vilarrubias (1994, 2000), en dos libros de Benito $(2000,2002)$ y un estudio terminológico en un libro de Otero (2000).

Comprobado que no existe ningún Glosario científico de protocolo decidimos crear uno y para ello emplearemos el análisis de contenido primeramente, y más tarde el análisis del discurso.

La muestra que emplearemos en dicho análisis la obtenemos de un estudio documental bibliográfico y de un estudio autorial del protocolo. De este modo mediante un análisis de contenido a la documentación del protocolo que cumplía los requisitos de: ser monografías centradas en el protocolo y ceremonial, estar escritas por españoles, publicadas en España, hasta el año 2006; logramos acotar el campo de estudio desde más de 500 libros a sólo 170 escritos por 90 autores. Y posteriormente mediante tres estudios que investigan la cantidad de producción bibliográfica de cada autor, la citación de autores entre ellos y la reputación de los autores más importantes en España, obtenemos la lista de autoridades de protocolo que son autores de prestigio y crédito reconocido por su competencia en esta materia que elaboran textos de calidad, válidos y relevantes. 
De este modo, comparando resultados obtuvimos a 6 autoridades que nos aportan los textos fundantes del protocolo para continuar con la investigación que son 35: Felio A. Vilarrubias Solanes (1976, 1980, 1991, 1994, 1997, 2000, 2003, 2004, 2005), Francisco López-Nieto y Mallo (1985, 1995, 1997, 1998, 1999, 2000a, 2000b, 2003a, 2003b), José Antonio de Urbina (1989, 1991, 1994, 1995, 2000, 2001, 2002, 2004), Carlos Fuente Lafuente (2004a, 2004b, 2004c, 2005), Francisco Marín Calahorro (1997, 2000, 2004) y María Teresa Otero Alvarado (1999, 2000, 2001).

\section{Palabras más definidas}

Una vez que tenemos la muestra acotada buscamos el lenguaje y los conocimientos comúnmente aceptados por el grupo profesional y académico del protocolo.

El léxico son las piezas de representación lingüística en las que debe apoyarse cualquier campo de conocimiento científico para poder adquirir, generar y transferir el conocimiento específico de cualquier dominio (Cabré, 1999: 70). Eso quiere decir que en cualquier campo científico la terminología desempeña un papel fundamental como proveedora de términos transportadores de conocimiento que median en la comunicación, como identificador de reglas subyacentes en la generación y relación de los términos, y como método y habilidad de trabajo.

Además, y como sucede en otros campos, el léxico se trata de uno de los factores que dificulta o ralentiza la elaboración de un cuerpo doctrinal homogéneo sobre protocolo. La diversidad conceptual del protocolo, en relación con ofrecer una definición que sirva de marco de referencia y que aporte el conocimiento necesario al receptor, constituye uno de los mayores obstáculos para el desarrollo de un cuerpo teórico sólido de la disciplina.

Por todo ello, efectuamos un análisis léxico del protocolo en sus autoridades, y en concreto, a través del análisis de contenido (Krippendorff, 1991: 40-44, Berganza y Ruiz, 2005: 217 y Berelson, 1952, 1959: 1-6). El objetivo de este análisis es estudiar los términos clave y los conceptos básicos del protocolo elaborados personalmente por las autoridades que están contenidos en el corpus documental seleccionado. Para investigar el contenido realizamos dos trabajos sobre el campo documental que codificaremos y registraremos en tablas: primero un análisis de contenido cuantitativo, tanto comparativo como descriptivo de las definiciones creadas por las autoridades de protocolo; y después un análisis de contenido más cualitativo de las definiciones de los términos seleccionados para la propuesta de un glosario básico de protocolo (Romero, 1991). 


\begin{tabular}{|c|c|c|c|c|c|c|c|}
\hline Palabras más definidas & Vilarrubias & López & Urbina & Fuente & Marín & Otero & Total \\
\hline Protocolo & 16 & 8 & 7 & 6 & 4 & 10 & 51 \\
\hline Precedencia & 8 & 3 & 5 & & 3 & 1 & 20 \\
\hline Ceremonial & 4 & 1 & 2 & 1 & 2 & 4 & 14 \\
\hline Presidencia & 5 & 3 & 3 & 2 & 1 & & 14 \\
\hline Etiqueta & 5 & 1 & & 1 & & 5 & 12 \\
\hline Peinado & 1 & 1 & 1 & 4 & 1 & & 8 \\
\hline Cortesía & 3 & & 4 & & & & 7 \\
\hline Anfitrión & 5 & & & 1 & 1 & & 7 \\
\hline Condecoraciones & 2 & 1 & 2 & & 2 & & 7 \\
\hline Cronograma & 1 & 1 & & 3 & 2 & & 7 \\
\hline Tratamiento & 1 & 2 & 3 & & 1 & & 7 \\
\hline Acto & & & 1 & 3 & & & 4 \\
\hline Derecho premial & 3 & 1 & & & & & 4 \\
\hline Protocolo social & & 2 & 2 & & & & 4 \\
\hline
\end{tabular}

Tabla 1. Palabras más definidas detalladas por autoridad. Elaboración propia.

El resultado del análisis de contenido es un conjunto de 134 palabras definidas y 335 definiciones originales, lo que nos obliga a delimitar cuantitativamente los términos claves del protocolo español definidos por sus propias autoridades a una selección de sólo 14, los más definidos.

\section{Palabras más definidas y sus definiciones}

Siguiendo el análisis de contenido frecuencial bajo principios semióticos iniciado en el punto anterior que contabiliza las palabras más definidas, las clasifica y las numera según su importancia, procedemos a iniciar el análisis de significados y la interpretación, que estudian el sentido y el valor de las palabras definidas para conocer su significado (Romero, 1991).

De aquí en adelante utilizaremos como unidades a analizar e interpretar, el grupo de las palabras más definidas por las autoridades, para así estudiar la amplitud y profundidad de cada definición y sus relaciones con otras similares o cercanas. Con esta fase investigamos los matices de cada definición propuesta para las palabras claves del protocolo de modo: individual, cuantitativo, cualitativo y objetivo, para así plantear y discutir cual es la definición más acertada para cada término raíz del protocolo.

Toda la información obtenida anteriormente la clasificamos siguiendo la técnica para definir de Ackoff (Sierra Bravo, 2002: 80), lo que nos permite un análisis individual de cada categoría que muestra un listado de definiciones agrupadas por autoridad, y varios comentarios que analizan e interpretan los datos. Para realizar los análisis comparativos y descriptivos de las definiciones de las autoridades 
aportadas para cada término se sigue un criterio de actualidad, antigüedad, repetición, precisión y claridad. Pero debido al volumen de definiciones primero se analizan las definiciones de un término aportadas por la misma autoridad para así seleccionar la mejor de ellas y compararla, en una segunda etapa, con las seleccionadas de esa forma de las demás autoridades.

El Glosario Final presenta las definiciones originales recopiladas de las distintas fuentes bibliográficas. Cada noción se analiza y compara para acercarnos en lo posible a la esencia de cada concepto. En beneficio de la claridad y sin sacrificar el rigor, se pretende que las definiciones resulten inteligibles y comprensibles para los iniciados en la especialidad. Incluso podemos decir que su estructura redaccional es similar al de la cita bibliográfica ya que el texto definitorio va precedido por el libro donde se encuentra, codificado mediante su fecha de publicación, y la página donde se localiza.

Así, con este procedimiento de comparación y análisis, perseguimos esa definición ideal, es decir, al significado formado por los rasgos más relevantes del concepto aportados por las autoridades que constituirán el Glosario de Protocolo.

Una aproximación al Glosario desvela dificultades para definir de modo unívoco determinados conceptos, al contar con palabras que presentan múltiples definiciones con grandes diferencias conceptuales entre sí. Como ejemplo podemos mencionar el término protocolo que posee 51 definiciones distintas aportadas entre las seis autoridades, lo que también implica la imposibilidad de citarlas en este artículo debido a su volumen.

Por ello tras realizar sistemática y metódicamente el análisis individual y objetivo de cada definición atribuida a una de las catorce palabras más importantes de la terminología del protocolo, según las autoridades, presentamos por fin un listado de palabras relativas al protocolo y el ceremonial con una única definición para cada una, excepto cinco. Los términos protocolo, precedencia, ceremonial, etiqueta, acto y protocolo social, no presentan en este listado una definición debido a problemas particulares que poseen, de manera que el glosario está todavía incompleto y demanda un cambio metodológico para finalizarlo.

\section{Algunas palabras clave del Protocolo}

Tras observar los análisis cuantitativos realizados con anterioridad constatamos que hay cinco palabras nucleares del protocolo que son las más definidas por las autoridades, así como las más problemáticas para estudiar mediante análisis de contenido debido a la variedad, la diferencia, la complejidad y la extensión de las definiciones aportadas para cada una de ellas. Así mismo la palabra acto, necesita un mayor análisis definitorio, y protocolo social, debe esclarecer su independencia del término protocolo. 
De este modo los términos protocolo, precedencia, ceremonial y etiqueta que no obtuvieron definición alguna, junto a acto y protocolo social que no presentan definiciones muy claras, motivan el abandono del análisis de contenido clásico y la teoría semiótica, que ha dado sus frutos al lograr crear un léxico específico del protocolo compuesto por nueve términos, pero que ha sido incapaz de completarlo en su totalidad.

Como ya se ha constatado a lo largo de estas páginas, el protocolo es un fenómeno complejo por lo que definirlo no es tarea nada sencilla. En cuanto sector de la realidad social, se comprueba claramente que ha sido objeto de numerosas definiciones con alcances y características muy distintas. A lo largo de nuestro análisis de las diferentes concepciones teóricas que le afectan, hemos advertido ya esa variedad de confines conceptuales. Sin embargo, a diferencia de otras disciplinas, se añade el factor ya aludido de la vulgarización y ambigüedad del concepto, lo que complica todavía más el panorama.

El protocolo tiene numerosas denominaciones que varían según las características del texto del que son extraídas por lo que se encuentran múltiples formas de referirse a la disciplina. Es importante tener presente esta variación en la denominación para así poder circunscribir nuestro análisis a todas las formas relevantes de denominación de un concepto, sin dejar de lado ninguna. Puesto que nuestro análisis es descriptivo, no podemos dejar de ofrecer todas las formas encontradas.

La gran dificultad de realizar el análisis conceptual a todos los términos claves del protocolo a través de un análisis de contenido, motiva que se incorporen otras técnicas a la investigación relacionadas con el análisis del discurso (Teun van Dijk: 2000a, 2000b) para así interpretar en una aproximación más hermenéutica las propuestas de definición del léxico del protocolo obtenidas de los textos autoriales y mediante la representación gráfica de la semántica de cada término problemático gracias al empleo de mapas conceptuales.

De esta forma, el análisis individual de cada término se compone por un listado de definiciones agrupadas por autoridad, varios comentarios que analizan e interpretan los datos y diversos mapas conceptuales. Debido a la existencia de muchas definiciones para el mismo término, incluso varias de la misma autoridad, tenemos que aplicar técnicas comparativas poniendo en correspondencia unas definiciones con otras, para ver sus diferencias y semejanzas. De ese modo, para realizar el análisis y la interpretación de la definición individual de cada término, tenemos que cumplir varios criterios comparativos en este orden: la actualidad de la definición, la antigüedad de la definición, la repetición de elementos dentro de las definiciones, la precisión de la definición y la claridad de la definición.

Precisamente, debido a la ordenación de las definiciones en este análisis, se observará la evolución conceptual de cada término en función de las autoridades por lo que veremos definiciones muy similares con el paso del tiempo pero, con alguna idea nueva, mayor o menor especificidad, etc. 


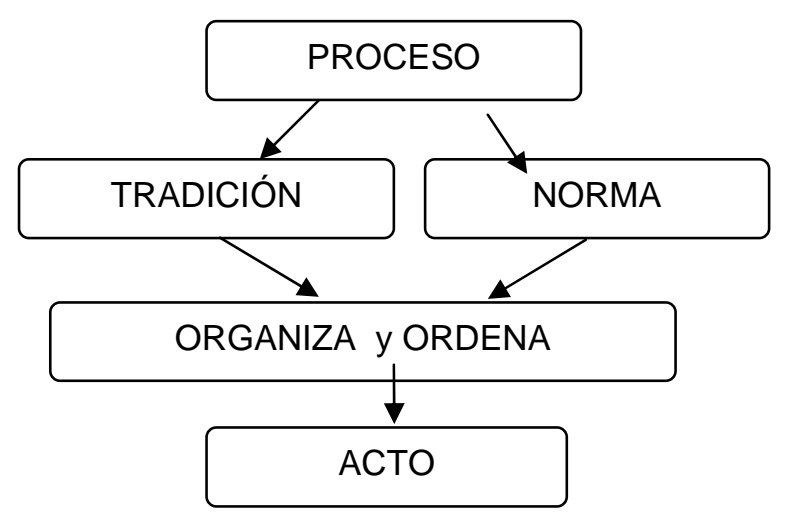

Figura 1. Mapa conceptual de protocolo. Elaboración propia.

\section{Glosario de Protocolo}

Finalmente los análisis determinan que el Glosario se compone por un total de trece términos junto a sus definiciones que representan a la disciplina en la bibliografía documental de las autoridades españolas señaladas, lo que los convierte en el léxico indispensables a la hora de hablar de Protocolo, de modo que siempre que se emplee alguno de ellos, nos estaremos refiriendo a ésta (Álvarez, 2007).

Elaborar este glosario confirma las dificultades anteriormente citadas y revela la falta de acuerdo común entre las autoridades con respecto a las palabras claves del protocolo por lo que no debe extrañar que ese “caos conceptual” se refleje en la sociedad donde se desconoce profundamente a la disciplina. Por ello en varios términos aportamos nuestras propuestas conceptuales basadas en las ideas capitales aportadas por las autoridades, configurando así un Glosario con definiciones de las autoridades junto a varias nuevas propuestas léxicas.

1. Protocolo: Proceso tradicional regulado que planifica, ordena, organiza e investiga el acto público.

2. Precedencia: Ordenamiento espacial y temporal de los agentes participantes en un acto.

3. Ceremonial: Conjunto de formas para actos solemnes.

4. Presidencia: Vilarrubias (2004: 58) (2000: 45), Puesto de honor en todo acto que centra la atención de los invitados y de los medios de comunicación social, e indica quién ha de dirigir su desarrollo entre dos tiempos: el inicio y el final.

5. Etiqueta: Conjunto de formas para relaciones solemnes. 
6. Peinado: Vilarrubias (2000: 381) (1994: 322), Operación de interrelación de invitados procedentes de varias listas en una sola, por ordenación jerárquica.

7. Cortesía: Urbina (2004: 22) (2000: 48) (2001: 91) (1995: 48), Es la suma de respeto, tolerancia, amabilidad y afabilidad.

8. Anfitrión: Vilarrubias (2005: 21), Figura principal con puesto de honor, al que le corresponde dirigir la presidencia, las palabras de bienvenida y el discurso de clausura o apertura según la clase de acto.

9. Condecoraciones: Urbina (2000: 425) (1995: 425), Alta dignidad honorífica, personal e intransferible, otorgada por un jefe de Estado a una persona física, nacional o extranjera, o a una entidad, institución o persona jurídica en reconocimiento de sus importantes servicios a la nación.

10. Cronograma: Vilarrubias (2000: 377) (1994: 318), Correlación tiempo-espacio de un acto de protocolo.

11. Tratamiento: Vilarrubias (2004: 42) (2000: 32) (1994: 24), Trato determinado a cada persona con carácter honorífico, yendo unidos al rango del beneficiario, o a título personal, pudiendo tener condición temporal o permanente (es decir, con carácter vitalicio) y varios medios de utilización verbal o escrita.

12. Acto: Organización y dirección de determinados actos públicos que por su relevancia pretenden trasladar una comunicación a sus invitados y a sus espectadores.

13. Derecho Premial: Vilarrubias (2005: 25) (2004: 38) (1997: 25) (1991: 27), Reconocimiento jurídico de los actos meritorios a favor de la Comunidad o de un colectivo.

\section{Conclusiones}

Esta investigación se propuso conocer y esclarecer el léxico clave del protocolo y ceremonial debido a la importancia que reviste en cualquier materia, ya que conforma y transmite ciencia, pero principalmente por la problemática específica en la que se encuentra el protocolo.

Con este Glosario de Protocolo logramos reunir materiales hasta el momento dispersos en multitud de contenidos textuales de obras, y facilitamos además nuevas síntesis de los mismos que permitirán su uso práctico a la hora de estudiar y hablar sobre protocolo, y favorecer un mejor conocimiento de la fundamentación de esta materia a través de su terminología específica para así establecer una base científica para una posterior elaboración de un cuerpo teórico del protocolo.

El estudio del léxico del protocolo también nos ha mostrado la existencia de tres perspectivas teóricas muy marcadas en las diferentes autoridades a través de sus definiciones. Todos los conceptos analizados están pensados bajo alguna de estas tres perspectivas: histórica, jurídica y 
comunicológica; lo que indica un intento de reflexión epistemológica e investigadora entorno a la materia, así como un ordenamiento del conocimiento en cuatro cuerpos doctrinales, de mayor o menor contenido teórico que podemos denominar escuelas.

No obstante, como se ha podido ver a lo largo de los diversos estudios, el protocolo todavía no presenta unos conceptos comunes lo que nos ha obligado a realizar varias propuestas basadas en el conjunto de ideas de las autoridades. De este modo nuestro Glosario de protocolo no sólo pretende exponer y dar a conocer el léxico de esta materia sino que busca el debate, tanto de la comunidad académica como de los expertos y profesionales del protocolo, que motive un apoyo o refutación a los elementos del Glosario recogidos en este artículo.

Sin embargo en un futuro, para obtener las definiciones básicas del protocolo, quizá se pueda realizar un análisis del discurso protocolario, investigando, en lugar de una amplia selección de documentación bibliográfica, un conjunto de discursos narrativos creados por los actos protocolarios, donde la representación simbólica y la participación activa de los públicos puedan ser más determinantes en la definición y evaluación de resultados.

\section{Bibliografía}

- ÁLVAREZ, M. L.; 2008a: Nociones de protocolo desde la bibliografía de sus autoridades, Tesis doctoral dirigida por Daniel Martí. Universidad de Vigo, Facultad de Ciencias Sociales y de la Comunicación, Departamento de Psicología Evolutiva y Comunicación.

- ÁLVAREZ, M. L.; 2008b: Nociones de protocolo desde la bibliografía de sus autoridades, en Revista Latina de Comunicación Social, 63, pp. 165-173. La Laguna (Tenerife): Universidad de La Laguna, consultado el 1-4-2008, en http://www.ull.es/publicaciones/latina/_2008/15_08_Vigo/ML_Alvarez_Rodriguez.html.

- ÁlvareZ, M. L.; 2008c: Protocolo y Comunicación, blog consultado el 1-4-2008, en http://www.protocoloycomunicacion.com

- CABRÉ, M. T.; 1999: La terminología. Representación y comunicación. Una teoría de base comunicativa y otros artículos. Barcelona, Iulaterm. 
- CLAUSÓ, A.; 1996: Manual de análisis documental: descripción bibliográfica, Pamplona, EUNSA.

- $\quad$ BARDIN, L.; 1986: Análisis de contenido, Madrid, Akal Universitaria.

- BUENO CAMPOS, E.; 1996: Organización de empresas: estructura, procesos y modelos, Madrid, Pirámide.

- BERELSON, B.; 1952: Content Analysis in Communication Research, Nueva York, Free Press.

- BERELSON, B.; 1959: The State of Communication Research. Public Opinion Quaterly, 23, pp. $1-6$.

- BERGANZA, M. R. y RUIZ, J. A.; 2005: Investigar en Comunicación, Madrid, McGraw Hill.

- DIJK, T. A. van; 2000a: El discurso como estructura y proceso, Barcelona, Gedisa.

- $\quad$ DIJK, T. A. van; 2000b: El discurso como interacción social, Barcelona, Gedisa.

- FUENTE LAFUENTE, C.; 2004a: La boda Real, Madrid, Ediciones Protocolo.

- FUENTE LAFUENTE, C.; 2004b: Protocolo oficial, Madrid, Ediciones Protocolo.

- FUENTE LAFUENTE, C.; 2004c: Técnicas de organización de actos, Madrid, Ediciones Protocolo.

- FUENTE LAFUENTE, C.; 2005: Técnicas de organización de actos II, Madrid, Ediciones Protocolo.

- HERNÁNDEZ MARTÍNEZ, S.; 2006: Los gabinetes de protocolo como herramienta de Relaciones Públicas en las universidades españolas en el siglo XXI. Tesis Doctoral dirigida por M ${ }^{\mathrm{a}}$ Teresa Otero Alvarado. Universidad de Sevilla, Facultad de Comunicación, Departamento de Comunicación Audiovisual, Publicidad y Literatura.

- HERnÁNDEZ MARTÍNEZ, S.; 2000: El Protocolo hoy, Sphera Pública, nº 0, pp. 187-195

- KRIPPENDORFF, K.; 1991: Metodología de análisis de contenido, Barcelona, Paidós.

- $\quad$ LÓPEZ-NIETO Y MALLO, F.; 1985: Honores y protocolo, Madrid, El Consultor.

- $\quad$ LÓPEZ-NIETO Y MALLO, F.; 1995: Manual de protocolo, Barcelona, Ariel. 
- LÓPEZ-NIETO Y MALLO, F.; 1997: Manual de protocolo, 2ª ed., Barcelona, Ariel.

- LÓPEZ-NIETO Y MALLO, F.; 1998: La documentación del protocolo, Madrid, Bayer Hermanos.

- LÓPEZ-NIETO Y MALLO, F.; 1999: Legislación de protocolo, Madrid, Dykinson.

- LÓPEZ-NIETO Y MALLO, F.; 2000a: Honores y protocolo, 2ª ed., 2 vols., Madrid, El Consultor.

- LÓPEZ-NIETO Y MALLO, F.; 2000b: Manual de protocolo, $3^{\mathrm{a}}$ ed., Barcelona, Ariel.

- LÓPEZ-NIETO Y MALLO, F.; 2003a: La secretaria y el protocolo, Madrid. Libertarias.

- LÓPEZ-NIETO Y MALLO, F.; 2003b: Manual de protocolo, 4ª ed., Barcelona, Ariel.

- MARÍN CALAHORRO, F.; 1997: Fundamentos del Protocolo en la Comunicación Institucional, Madrid, Síntesis.

- MARÍN CALAHORRO, F.; 2000: Protocolo y comunicación. Los medios en los actos públicos, Barcelona, Ediciones Bayer Hermanos.

- MARÍN CALAHORRO, F.; 2004: El protocolo en los actos de empresa, Madrid, Ediciones Fragua.

- PIÑUEL, J. L.; GAITÁN, J. A.; 1995: Metodología general. Conocimiento científico e investigación en la comunicación social, Madrid, Síntesis.

- Otero AlVARAdO, M. T.; 1999: Protocolo y Relaciones Públicas de Estado: Los Días Nacionales en la Exposición Universal de Sevilla 1992. Tesis doctoral dirigida por Juan Rey Fuentes. Universidad de Sevilla, Facultad de Ciencias de la Información, Departamento de Comunicación Audiovisual y Publicidad, Periodismo y Literatura.

- OTERO ALVARADO, M. T.; (2000): Teoría y estructura del ceremonial y el protocolo, Sevilla, Mergablum.

- Otero AlVARADO, M. T.; 2001: Relaciones Públicas y Protocolo: cinco años de reflexiones, Sevilla, Laurea. 
- OTERO ALVARADO, M. T.; 2002: Las funciones del ceremonial y el protocolo en la reputación corporativa, Sphera Pública: Organización y Cultura, la Identidad Corporativa, $\mathrm{n}^{0}$ 2, pp. 135-147.

- $\quad$ PORTO SIMOES, R.; 1993: Relaciones Públicas: función política. Barcelona, Consejo Superior de Relaciones Públicas de España.

- RODRIGO ALSINA, M.; 2001: Teorías de la Comunicación, Barcelona, Universidad Autónoma.

- ROMERO, A.; 1991: Metodologia de Análise de Conteúdo, Lisboa, Universidad Católica Portuguesa.

- SIERRA BRAVO, R.; 2002: Tesis doctorales y trabajos de investigación científica, Madrid, Paraninfo.

- SIERRA SÁNCHEZ, J.; 2007: Protocolo: símbolos y comunicación en el siglo XXI. Tesis doctoral dirigida por Francisco García García. Universidad Complutense de Madrid, Facultad de Ciencias de la Información, Departamento de Comunicación Audiovisual y Publicidad II.

- URBINA, J. A. de; 1989: El arte de invitar: su protocolo, Madrid, Consejo Superior de Comunicación y Relaciones Públicas de España.

- URBINA, J. A. de; 1991: Manual de protocolo diplomático español: para el uso de las Misiones Diplomáticas, sus Oficinas Consulares y los Organismos Internacionales acreditados en España, Madrid, Ministerio de Asuntos Exteriores.

- URBINA, J. A. de; 1995: El protocolo en casa: usos y costumbres, ideas y soluciones en nuestra vida cotidiana, Madrid, Temas de hoy.

- URBINA, J. A. de; 1996: El Protocolo en los negocios: las reglas de oro del saber ser, estar y funcionar..., Madrid, Temas de hoy.

- URBINA, J. A. de; 2000: El Protocolo en los negocios: las reglas de oro del saber ser, estar y funcionar..., Madrid, Temas de hoy. 
- URBINA, J. A. de; 2001: El Gran libro del protocolo, Madrid, Temas de hoy.

- $\quad$ URBINA, J. A. de; 2002: El Arte de invitar: su protocolo, Madrid, Temas de hoy.

- URBINA, J. A. de; 2004: 100 preguntas básicas de protocolo, Madrid, Temas de hoy.

- VILARRUBIAS SOLANES, F. A.; 1976: Introducción al protocolo y ceremonial en las corporaciones locales, Madrid, Instituto de estudios de la administración local.

- VILARRUBIAS SOLANES, F. A.; 1980: Honores, distinciones y heráldica en las corporaciones locales, Madrid, Instituto de estudios de la administración local.

- VILARRUBIAS SOLANES, F. A.; 1991: El protocolo en los actos de la administración, de las corporaciones y de las empresas, Oviedo, Universidad de Oviedo.

- VILARRUBIAS SOLANES, F. A.; 1994: Tratado de protocolo de estado e internacional, Oviedo, Nobel.

- VILARRUBIAS SOLANES, F. A.; 1997: Protocolo, ceremonial y heráldica de las corporaciones públicas y las empresas, $2^{\text {a }}$ ed., Oviedo, Universidad de Oviedo.

- VILARRUBIAS SOLANES, F. A.; 2000: Tratado de protocolo de estado e internacional, $2^{\mathrm{a}}$ ed., Oviedo, Nobel.

- VILARRUBIAS SOLANES, F. A.; 2003: La forma y el ser en el protocolo, ceremonial, heráldica y vexilología, Oviedo, Universidad de Oviedo.

- VILARRUBIAS SOLANES, F. A.; 2004: Tratado de protocolo, $3^{\mathrm{a}}$ ed., Oviedo, Nobel.

- VILARRUBIAS SOLANES, F. A.; 2005: Derecho Premial. Protocolo, Ceremonial, Heráldica y Vexilología en el Estado, en las corporaciones públicas y en la empresa multinacional, $3^{\mathrm{a}}$ ed., Oviedo, Universidad de Oviedo. 